\title{
Social Circles: A 3D User Interface for Facebook
}

\author{
Diego Rodrigues and Ian Oakley \\ Lab:USE, Department of Mathematics and Engineering, University of Madeira \\ Campus Universitário da Penteada, Funchal, 9000-390, Portugal \\ diegoajrodrigues@gmail.com, ian@uma.pt
}

\begin{abstract}
Online social network services are increasingly popular web applications which display large amounts of rich multimedia content: contacts, status updates, photos and event information. Arguing that this quantity of information overwhelms conventional user interfaces, this paper presents Social Circles, a rich interactive visualization designed to support real world users of social network services in everyday tasks such as keeping up with friends and organizing their network. It achieves this by using 3D UIs, fluid animations and a spatial metaphor to enable direct manipulation of a social network.
\end{abstract}

Keywords: Social network, visualization, direct manipulation, 3D UI, animation.

\section{Introduction}

Web 2.0 has ushered in an age of media rich, connected and highly interactive web applications. Among the most popular of these are online social networking services, such as Facebook (www.facebook.com) which boasts millions of users and hits per day. The appeal of online social networking services is clear: they enable users to easily form persistent networks of friends with whom they can interact and share content. However, online social network services have rapidly evolved into highly complex systems which contain a large amount of personally salient information derived from large networks of friends [1]. This paper argues that the amount of information presented in these systems exceeds the abilities of users to view and absorb it using traditional desktop user interfaces: it is slow, inefficient and frustrating. To address this issue, it describes the design of Social Circles, a rich visual interface focused on effectively presenting the key information that users require from social network services. This approach differs from previous work on the visualization of social networks which has been largely focused on analytical tasks and inspired by the diagramming conventions of social network theory [e.g. 2].

\section{Social Circles}

The design of Social Circles was fundamentally informed by the desire to place all the relevant content relating to a user's friends on a single screen. The overall goal is to enable an at-a-glance overview of activity in the entire network and rapid, seamless navigation to particular content items of interest. In order to achieve this, the system uses a spatial metaphor and the principles of direct manipulation. It argues that a spatial 

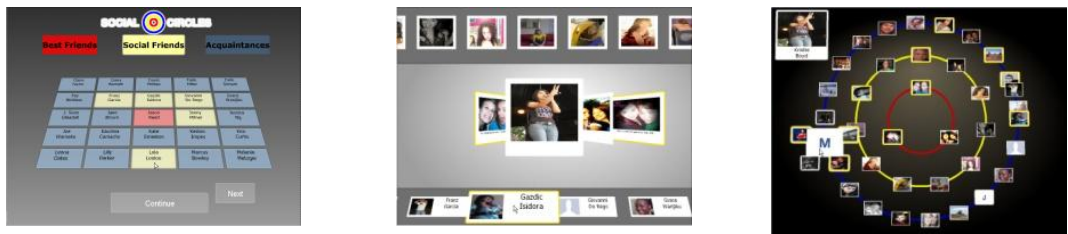

Fig. 1. a) User categorization UI (left), b) Rows UI (mid) and c) Circles UI (right)

metaphor can communicate important structural aspects of a user's network (such as the "social distance" of particular individuals, or the presence of particular salient groups and clusters) in a way that conventional UI techniques, involving the largely textual construction of lists or groups, cannot. Furthermore, an animated spatial interface supports direct interactions in the form of not only selection, but also hovering over items to highlight and dragging content to alter properties and change configurations.

Reflecting these motivations, the Social Circles UI represents a user's friends as visual icons resembling name cards (or Polaroid photos) arranged in 3D space. It allows users to classify friends according to three levels of social distance (best friends, social friends and acquaintances) and illustrates this distinction spatially. Two arrangements are shown in Figure 1, one based on differently sized cards arranged in three horizontal rows and the other on cards in three concentric circles. In the former UI, card size represents social distance while in the later it is the radius of the circle. In both interfaces, users can promote and demote friends simply by selecting and dragging them to desired new level. The cards also display extra information about the friends they depict. A border to the cards (shown yellow in the figures) and conspicuous pictorial icons highlight friends with recently updated status text or photo albums. Hovering over a card causes it to expand in place and reveal a friend's name; clicking causes it to move to the center of the screen and show the most recent text status. The card can then be clicked again to display a full screen profile page of the friend, or the close widget (in the corner of a card) can be selected to collapse the card back down to its default presentation. The interfaces differ in their presentation of the dense social network content: the rows UI uses a carousal metaphor which scrolls content in response to user input, whereas the circular UI clusters friends into alphabetic groups which expand when the cursor hovers over them. We are currently exploring the usability and scalability of these different design choices.

Social Circles is implemented as a cross-platform Facebook application using Adobe Flash, the Papervision3D engine and the GreenSock tweening platform.

In conclusion, this paper introduced Social Circles, a rich user interface for online social network services using a spatial metaphor, 3D rendering and animation. Future development of this work will include adding an interface for photo exploration and subjective and objective user study. We will also release a public beta of Social Circles to assess the real world applicability of this interface, design and approach.

\section{References}

1. Joinson, A.N.: Looking at, looking up or keeping up with people?: motives and use of facebook. In: Procs. CHI 2008 (2008)

2. Perer, A., Shneiderman, B.: Integrating Statistics and Visualization: Case Studies of Gaining Clarity during Exploratory Data Analysis. In: Procs of CHI 2008 (2008) 